\title{
Application of Soft Computing Paradigm to Large Deformation Analysis of Cantilever Beam under Point Load
}

\author{
Yanmei Cui, ${ }^{1}$ Yong Hong $\mathbb{D}^{1},{ }^{1}$ Naveed Ahmad Khan $\mathbb{D}^{2},{ }^{2}$ and Muhammad Sulaiman $\mathbb{D}^{2}$ \\ ${ }^{1}$ School of Mechanical Engineering, Shanghai Dianji University, Shanghai 201306, China \\ ${ }^{2}$ Department of Mathematics, Abdul Wali Khan University, Mardan, Khyber Pakhtunkhwa, Pakistan \\ Correspondence should be addressed to Yong Hong; hongyong83@126.com
}

Received 14 June 2021; Revised 19 July 2021; Accepted 5 November 2021; Published 29 November 2021

Academic Editor: Haitham Afan

Copyright (c) 2021 Yanmei Cui et al. This is an open access article distributed under the Creative Commons Attribution License, which permits unrestricted use, distribution, and reproduction in any medium, provided the original work is properly cited.

In this paper, a mathematical model for large deformation of a cantilever beam subjected to tip-concentrated load is presented. The model is governed by nonlinear differential equations. Large deformation of a cantilever beam has number of applications is structural engineering. Since finding an exact solution to such nonlinear models is difficult task, this paper focuses on developing soft computing technique based on artificial neural networks (ANNs), generalized normal distribution optimization (GNDO) algorithm, and sequential quadratic programming (SQP). The strength of ANN modeling for governing the equation of cantilever beam is exploited by the global search ability of GNDO and further explored by the local search mechanism of SQP. Design scheme is evaluated for different cases depending on variations in dimensionless end-point load $(\rho)$. Furthermore, to validate the effectiveness and convergence of algorithm proposed technique, the results of the differential transformation method (DTM) and exact solutions are compared. The statistical analysis of performance indicators in terms of mean, median, and standard deviations further establishes the worth of ANN-GNDO-SQP algorithm.

\section{Introduction}

Mechanical systems, which involve nonlinearity due to large deflection of compliant mechanism, continue to be an interesting problem. Since large deformation phenomena occur often in various geotechnical practices [1]. Therefore, this topic is of practical interest and has been widely studied by many researchers. In past decades, a number of numerical methods have been developed to solve large deformation problems. The large deflection of cantilever beams was studied by Wang [2]. They developed nonlinear differential equations for postbuckling loads on the basis of Eringen's nonlocal constitutive relation. Shooting method was used to obtain postbuckling load and the buckled shape of the beam. Framework of arbitrary Lagrangian-Eulerian (ALE) is commonly used approach to study large deformation in geotechnical engineering [3]. Based on ALE three different approaches named as the efficient ALE approach (EALE) $[4,5]$, interpolation technique by small strain (RITSS) $[6,7]$ and the Abaqus built-in coupled Eulerian-Lagrangian (CEL) method [8]. Modified Chebyshev's polynomial is used by Schmidt and Dadeppo [9] to study large deflection of beam. Large deformation of a spring-hinged beam was investigated by Nageswara Rao $[10,11]$ subjected to a tip rotational concentrated and distributed load.

Ludwick's large deformation was studied by Lee [12] to made cantilever beams with a combined loading effect of a focused load at the tip and a uniformly dispersed load over the beam length. Phungpaigram and Chucheepsakul $[13,14]$ used elliptic integrals to calculate exact solutions for large deflection in elastic beams with variations in arc length and inclined force. Dado and Al-Sadder $[15,16]$ investigated the behaviour of large deformation of prismatic and nonprismatic cantilever beams under various type of loadings. Wang $[17,18]$ used homotopy perturbation method (HPM) to obtain analytical solution for large deformation of beam under point load with free tip. References $[19,20]$ studied the longitudinal vibration analysis for microbars based on strain gradient elasticity theory. Feasibility of Adomian decomposition method for such complex nonlinear problems was 
studied by Tolou and Herder [21]. Mutyalarao [22] studied large deflections of a uniform cantilever beam with concentrated load at tip and having normal inclination to the deflected axis of the beam. All these recently introduced techniques have their own grains and limitations in terms of accuracy, robustness, convergence, and applicability, but they are based on well-established deterministic procedures. Complex nonlinear differential equation of large deformation of beams subjected to a concentrated load is of great interest in scientific and engineering field. The quest of finding analytical solution for the problem motivates the author to develop a soft computing technique based on feed forward artificial neural networks (ANNs).

Stochastic solvers based on computational intelligence methods using artificial neural networks (ANNs) are considered to be fundamental in pattern recognition and machine learning. In general, neural networks are widely used to solve fractional differential equations, integrodifferential equations (IDEs), partial differential equations (PDEs), and ordinary differential equations (ODEs). In ANN modeling, the optimization procedure is carried by using combination of global and local search algorithms. Some recent application of stochastic algorithm are multiphase flow through porous media for imbibition phenomena [23], eye model [24], wire coating dynamics $[25,26]$, optimal design and temperature distribution of heat fin $[27,28]$, beam-column design [29], and hybrid feature analysis for diabetic retinopathy classification using fundus images [30].

In the present study, a novel soft computing technique is applied to find analytical series solution for large deformation of cantilever beam subjected to point load by using ANN models optimized globally with generalized normal distribution optimization (GNDO) algorithm hybrid with sequential quadratic programming (SQP) for rapid local convergence. The prominent features of the present study are given as follows.

(i) Mathematical model for large deformation of cantilever beam under end point load is formulated and analyzed to study the influence of variations in dimensionless end point load $(\rho)$.

(ii) A novel soft computing paradigm is developed to model series solutions based on artificial neural networks with the generalized normal distribution optimization algorithm and the sequential quadratic programming. Our approach is named as ANNGNDO-SQP algorithm.

(iii) To validate the efficiency of the proposed technique, four cases of large deformation cantilever beam are considered. The statistical results are compared with differential transformation method (DTM) and analytical solutions.

(iv) Statistical analysis of absolute errors (AE), fitness evaluation (Fit), mean absolute deviation (MAD), Theil's inequality coefficient (TIC), root mean square error (RMSE), Nash-Sutcliffe efficiency (NSE), and error in Nash-Sutcliffe efficiency
(ENSE) are presented in terms of minimum, mean, median, and standard deviation.

(v) Provision of continuous solutions, computational complexity, convergence, and easy execution of the proposed methodology show the robustness and correctness of the ANN-GNDO-SQP algorithm.

\section{Problem Formulation}

Consider a cantilever beam with a large deformation due to end point load as shown is Figure 1. By Euler-Bernoulli beam theory, the curvature $(\kappa)$ of the beam can be given as

$$
\kappa=\frac{\mathrm{d} \theta}{\mathrm{d} s}=\frac{M}{\mathrm{EI}},
$$

where $\theta$ represents the slope or rotation of a beam, $s$ is the natural distance from the fixed end, $E$ is Young's modulus, $M$ is bending moment, $I$ is moment of inertia, and $\mathrm{EI}$ is the bending stiffness of cantilever beam. From Figure 1, moment $(M)$ for the deflected beam under end point load is given as

$$
M=F\left(L-\delta_{h}-x\right),
$$

where concentrated load at end point is denoted by $F, \delta_{h}$ is the horizontal deflection, and $L$ is the distance of deflected beam from fixed point. Hence, bending equation of uniform cross sectional beam for large deformation is written as

$$
\frac{\mathrm{d} \theta}{\mathrm{d} s}=\frac{F}{\mathrm{EI}}\left(L-\delta_{h}-x\right), \quad \theta(0)=0, \theta^{\prime}(L)=0 .
$$

If $F=0$, then concentrated force is a dead force such as gravity, and if $F=1$, then the concentrated force is perpendicular to the deflected beam at the end [31] (Figure 1).

Differentiation of (1) with respect to $s$ is given as

$$
\frac{\mathrm{d}^{2} \theta}{\mathrm{d} s^{2}}=\frac{\mathrm{d} M / \mathrm{d} s}{\mathrm{EI}},
$$

considering a dimensionless parameter $\xi=s / L$. Differentiating (2) with respect to $s$ and substituting $\cos (\theta)=\mathrm{d} x / \mathrm{d} s$ in (4) will result in governing second-order differential equation for large deformation of continuer beam with end point load as

$$
\frac{\mathrm{d}^{2} \theta}{\mathrm{d} \xi^{2}}+\rho \cos \theta=0
$$

with boundary conditions

$$
\begin{aligned}
& \text { at } \quad \xi=0, \theta=0, \\
& \text { at } \xi=1, \frac{\mathrm{d} \theta}{\mathrm{d} \xi}=0,
\end{aligned}
$$

where $\rho=\mathrm{FL}^{2} / \mathrm{EI}$, and it represents the dimensionless load at end point. Angle of rotation of the beam at free end is denoted by $\theta_{\text {tip }}(1)=1$. Furthermore, dimensionless horizontal displacement $\left(\delta_{h}\right)$ of the free end is given by $[17,32,33]$ as 


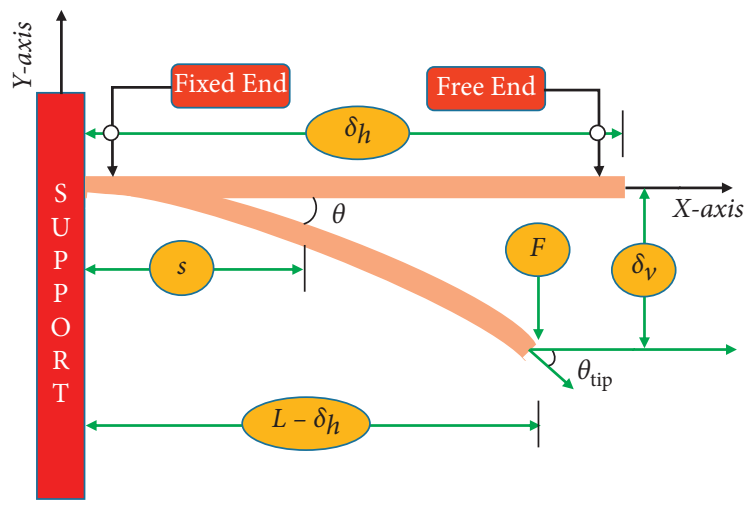

FIgURE 1: Large deformation of a cantilever beam under end point loading.

$$
\frac{L-\delta_{h}}{L}=\sqrt{\frac{2 \mathrm{EI}\left(\sin \theta_{\text {tip }}\right)}{\mathrm{FL}^{2}}}=\sqrt{\frac{2 \sin \theta_{\text {tip }}}{\rho}}
$$

then, the dimensionless horizontal displacement of the free tip is

$$
\frac{\delta_{h}}{L}=1-\frac{1}{\sqrt{\rho}} \sqrt{2 \sin \theta_{\text {tip }}} .
$$

For large deformation equation (5) along with boundary conditions, (6) and (7) are given as

$$
\begin{aligned}
\frac{\mathrm{d}^{2} \theta}{\mathrm{d} \xi^{2}}+\rho & =0, \\
\text { at } \quad \xi & =0, \theta=0, \\
\text { at } \quad \xi & =1, \frac{\mathrm{d} \theta}{\mathrm{d} \xi}=0 .
\end{aligned}
$$

The analytical solution for the problem is given as

$$
\theta(\xi)=\frac{\rho}{2}(2-\xi) \xi
$$

at the tip $\xi=1$.

$$
\theta(\xi)=\frac{\rho}{2}
$$

\section{Proposed Methodology}

The proposed soft computing paradigm for calculating approximate solutions for mathematical model of large deformation of cantilever beam consists of two parts. In the first part, an unsupervised ANNs model is constructed in terms of input, hidden, and output layers for governing differential equation. In the second part, neurons in ANN structure are trained or tuned by hybridizing generalized normal distribution optimization (GNDO) algorithm and sequential quadratic programming (SQP).

3.1. Construction of ANN Model. Feedforward artificial neural networks (ANNs) are used to model series solutions for governing equation of large deformation of cantilever beam with end point loading. Neural network model for (5)-(7) are formulated using continuous mapping approach for the solution $\theta(\xi)$ and its respective derivatives in terms of input, hidden, and output layer are given as follows:

$$
\begin{gathered}
\widehat{\theta}(\xi)=\sum_{i=1}^{k} \phi_{i} f\left(\omega_{i} \xi+\beta_{i}\right), \\
\hat{\theta}^{\prime}(\xi)=\sum_{i=1}^{k} \phi_{i} f^{\prime}\left(\omega_{i} \xi+\beta_{i}\right), \\
\hat{\theta}^{\prime \prime}(\xi)=\sum_{i=1}^{k} \phi_{i} f^{\prime \prime}\left(\omega_{i} \xi+\beta_{i}\right),
\end{gathered}
$$

where $\phi=\left[\phi_{1}, \phi_{2}, \phi_{3}, \ldots, \phi_{m}\right], \omega=\left[\omega_{1}, \omega_{2}, \omega_{3}, \ldots, \omega_{m}\right]$, and $\beta=\left[\beta_{1}, \beta_{2}, \beta_{3}, \ldots, \beta_{m}\right]$ are real-valued vectors and are bounded, $f$ is the activation function, and $i$ represents the number of neurons in ANN structure. In the hidden layer, (13)-(15) used log sigmoid as an activation function, and then, the updated form of solution and its derivatives is given as

$$
\begin{aligned}
\hat{\theta}(\xi) & =\sum_{i=1}^{k} \phi_{i} \omega_{i}\left(\frac{1}{1+e^{-\left(\omega_{i} \xi+\beta_{i}\right)}}\right), \\
\hat{\theta}^{\prime}(\xi) & =\sum_{i=1}^{k} \phi_{i} \omega_{i}\left(\frac{e^{-\left(\omega_{i} \xi+\beta_{i}\right)}}{\left(1+e^{-\left(\omega_{i} \xi+\beta_{i}\right)}\right)^{2}}\right), \\
\hat{\theta}^{\prime \prime}(\xi) & =\sum_{i=1}^{k} \phi_{i} \omega_{i}^{2}\left(\frac{2 e^{-\left(\omega_{i} \xi+\beta_{i}\right)}}{\left(1+e^{-\left(\omega_{i} \xi+\beta_{i}\right)}\right)^{3}}-\frac{e^{-\left(\omega_{i} \xi+\beta_{i}\right)}}{\left(1+e^{-\left(\omega_{i} \xi+\beta_{i}\right)}\right)^{2}}\right) .
\end{aligned}
$$

3.2. Formulation of Fitness Function. Fitness function or objective function for mathematical model of large deformation in cantilever beam is developed as a sum of two mean square error (MSE): 


$$
\text { Minimize } E=E_{1}+E_{2} \text {, }
$$

where $E_{1}$ and $E_{2}$ are MSE of (5) and (6) and (7), respectively, which are defined as

$$
\begin{aligned}
& E_{1}=\frac{1}{N} \sum_{m=1}^{N}\left(\frac{\mathrm{d}^{2} \widehat{\theta}}{\mathrm{d} \xi^{2}}+\rho\right)^{2} \\
& E_{2}=\frac{1}{2}\left((\widehat{\theta}(0)-0)^{2}+\left(\frac{\mathrm{d} \widehat{\theta}(1)}{\mathrm{d} \xi}-0\right)^{2}\right) .
\end{aligned}
$$

For the approximate solution of (5), fitness function equation (17) is optimized by training neurons in such a way that MSEs, $E_{1}$ and $E_{2}$, should approach to zero, and consequently, the approximate solution by proposed method will converge to exact solution.

3.3. Training of Neurons. Methodologies adopted for training of unknown neurons in ANNs structure for optimization of fitness function equation (17) are presented, which is based on hybridization of unsupervised and supervised learning of GNDO and SQP, respectively. The working mechanism of the proposed algorithm is provided in Figure 2.

3.4. Generalized Normal Distribution Optimization. Generalized normal distribution optimization (GNDO) algorithm is a novel metaheuristic technique presented by Zhang et al. [34], inspired by generalized normal distribution theory. GNDO algorithm is widely used for parameter extraction of model, unlike other metaheuristic algorithms; GNDO is easy to implement, and it only requires the essential population size and termination criteria. GNDO has a simple structure, where the position of each individual is updated by using normal distribution curve. The working strategy of GNDO algorithm is subdivided into two phases, exploitation and exploration.

3.4.1. Exploitation. Exploitation is a process of finding best solution around the search space consisting of the current positions of all individuals. Initially, model for optimization by generalized distribution model is given as

$$
v_{i}^{t}=\widehat{\mu}_{i}+\widehat{\delta}_{i} \times \widehat{\eta}, \quad i=1,2,3, \ldots, N
$$

where $v_{i}^{t}, \widehat{\mu}_{i}, \widehat{\delta}_{i}$, and $\widehat{\eta}$, are trial vector, generalized mean position, generalized standard variance, and penalty factor, respectively. Moreover, $\widehat{\eta}, \widehat{\delta}_{i}$, and $\widehat{\mu}_{i}$ are formulated as

$$
\begin{aligned}
& \hat{\eta}= \begin{cases}\sqrt{-\log \left(\zeta_{1}\right)} \times \cos \left(2 \pi \zeta_{2}\right), & \text { if } a \leq b, \\
\sqrt{-\log \left(\zeta_{1}\right)} \times \cos \left(2 \pi \zeta_{2}+\pi\right), & \text { otherwise, }\end{cases} \\
& \widehat{\delta}_{i}=\sqrt{\frac{1}{3}\left[\left(x_{i}^{t}-\widehat{\mu}\right)^{2}+\left(x_{\text {Best }}^{t}-\widehat{\mu}\right)^{2}+(M-\widehat{\mu})^{2}\right]} \\
& \widehat{\mu}_{i}=\frac{1}{3}\left(\mathbf{x}_{i}^{t}+\mathbf{x}_{\text {Best }}^{t}+M\right), \\
& M=\frac{\sum_{i=1}^{N} \mathbf{x}_{i}^{t}}{N}
\end{aligned}
$$

where $M$ is the mean position, $x_{\text {Best }}^{t}$ is the current best so far, and $a, b, \zeta_{1}$, and $\zeta_{2}$ are the random numbers between 0 and 1 . Furthermore, $\widehat{\eta}, \widehat{\delta}_{i}$, and $\widehat{\mu}_{i}$ are discussed in the exploration phase.

3.4.2. Exploration. Exploration refers to the searching of population space to get best solution. Exploration of GNDO is based on three randomly selected individuals as follows:

$$
\mathbf{v}_{i}^{t}=\mathbf{x}_{i}^{t}+\underbrace{\beta \times\left(\left|\zeta_{3}\right| \times \mathbf{v}_{1}\right)}_{\text {Local information sharing }}+\underbrace{(1-\beta) \times\left(\left|\zeta_{4}\right| \times \mathbf{v}_{2}\right)}_{\text {Global information sharing }},
$$

where $v_{1}$ and $v_{2}$ are the trail vectors, $\beta$ is the adjustment parameter, and $\zeta_{3}$ and $\zeta_{4}$ are the random numbers between 0 and 1 , which are subjected to the standard normal distribution. Trail vectors are computed as follows:

$$
\begin{aligned}
& \mathbf{v}_{1}= \begin{cases}\mathbf{x}_{i}^{t}-\mathbf{x}_{p 1}^{t}, & \text { if } f\left(\mathbf{x}_{i}^{t}\right)<f\left(\mathbf{x}_{p 1}^{t}\right), \\
\mathbf{x}_{p l}^{t}-\mathbf{x}_{i}^{t}, & \text { otherwise, }\end{cases} \\
& \mathbf{v}_{2}=\left\{\begin{array}{cl}
\mathbf{x}_{p 2}^{t}-\mathbf{x}_{p 3}^{t}, & \text { if } f\left(\mathbf{x}_{p 2}^{t}\right)<f\left(\mathbf{x}_{p 3}^{t}\right), \\
\mathbf{x}_{p 3}^{t}-\mathbf{x}_{p 2}^{t}, & \text { otherwise, }
\end{array}\right.
\end{aligned}
$$

where $p 1, p 2$, and $p 3$ are integers. It is worth mentioning that GNDO algorithm is inspired by the relationship between normal distribution law and traditional teaching phenomena, search process of metaheuristics, and group teaching phenomena, respectively. GNDO has been applied to study the parameter extraction of photovoltaic models [34].

3.5. Sequential Quadratic Programming. The best performance (weights) obtained by GNDO algorithm is refined by the process of hybridization with efficient local search technique known as sequential quadratic programming using MATLAB toolbox setting. SQP is one of the powerful methods for numerical solution of constrained 

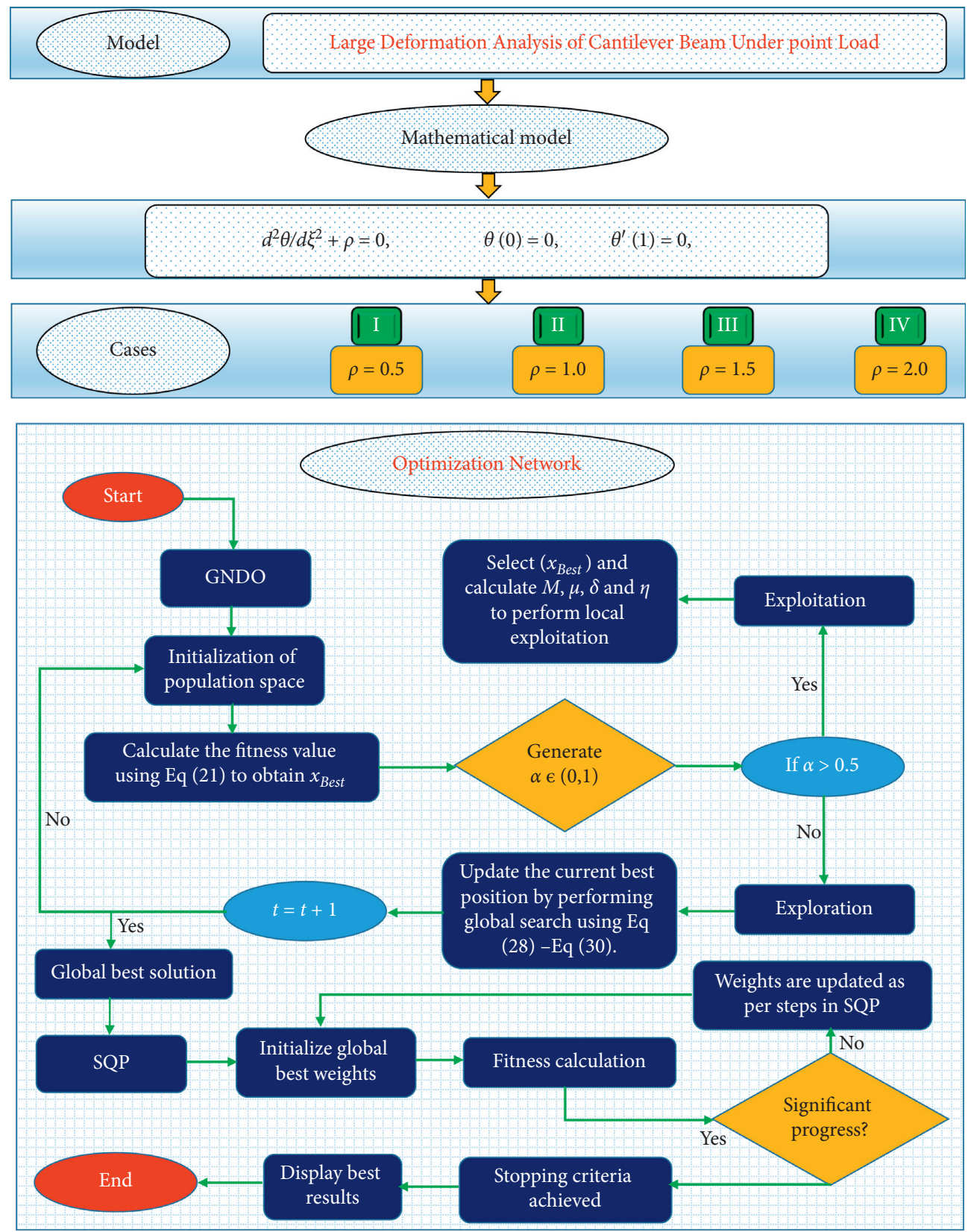

Figure 2: Graphical overview of the model, cases studies, and flow chart of the ANN-GNDO-SQP algorithm.

nonlinear optimization problems. It was developed in 1963 and further refined in 1970 [35]. SQP has been applied to a number of problems, which proves its power, accuracy, and efficiency. Nocedal and Wright [35] discuss SQP in detail and also give a mathematical formulation for various large-scale numerical optimization problems. Some recent applications of SQP are numerical solution for transient heat conduction problem [36], profile error evaluation of free-form surface [37], nonlinear model predictive control [38], OPF problem in DC grids [39], Bagley-Torvik systems arising in fluid mechanics [40], and optimal design of heating system in rapid thermal cycling blow mold [41].
3.6. Hybrid ANN-GNDO-SQP Algorithm. Necessary details of the procedural steps for proposed algorithm are given as follows.

Step 1. Initialization of GNDO: Initial weights are created randomly from population space with number of entries equal to number of neurons in ANN structure. Mathematical formulation is given as

$$
C=[(\phi, \omega, \beta)]^{T}=\left[\begin{array}{ccc}
\phi_{1} & \omega_{1} & \beta_{1} \\
\phi_{2} & \omega_{2} & \beta_{2} \\
\vdots & \vdots & \vdots \\
\phi_{m} & \omega_{m} & \beta_{m}
\end{array}\right],
$$


where $\phi, \omega$, and $\beta$ are the real values of unknown neurons in ANN model. Parameter setting for GNDO algorithm is given in Table 1.

Step 2. Fitness evaluation: Objective function equation (17) is evaluated to calculate the fitness value for the problem using the weights generated in the previous step.

Step 3. Criteria for termination: Execution of GNDO is stopped when any of the following criteria is satisfied.

Objective value ie $\varepsilon \longrightarrow 10^{-15}$

Function tolerance ie /Fun/TOL $\longrightarrow 10^{-15}$

Predefined number of iterations is achieved

If stopping criteria are fulfilled, then go to step $\mathrm{V}$ otherwise continue.

Step 4. Storage: Store the global best weight corresponding to minimum fitness value and time taken for the execution.

Step 5. Hybridization: Global best weights obtained by GNDO for minimization of (21) are considered as an initial guess for SQP to start the procedure.
Step 6. Fitness evaluation: SQP starts the supervised learning, update the weights, and evaluate the fitness function until the following terminations conditions are satisfied.

Objective value, i.e., $\varepsilon \longrightarrow 10^{-15}$.

Predefined number of iterations is achieved.

Step 7. Storage: Store the best weight, minimum fitness value, and time taken for the execution by SQP and the total time by GNDO-SQP in seconds.

Repeat the procedure from steps I-VII for a sufficient large number of independent runs to generate a large dataset for reliable statistical analysis.

\section{Performance Indices}

In this section, the performance of design scheme for solving mathematical model of large deformation of cantilever beam is examined by incorporating performance indicators in terms of mean absolute deviation (MAD), Theil's inequality coefficient (TIC), root mean square error (RMSE), and Nash-Sutcliffe efficiency (NSE). Mathematical formulations of these indicators are given as follows [23].

$$
\begin{aligned}
& \mathrm{MAD}=\frac{1}{N} \sum_{m=1}^{N}\left|\theta_{m}(\xi)-\widehat{\theta}_{m}(\xi)\right|, \\
& \mathrm{TIC}=\frac{\sqrt{(1 / N) \sum_{n=1}^{N}\left(\theta_{m}(\xi)-\hat{\theta}_{m}(\xi)\right)^{2}}}{\left(\sqrt{(1 / N) \sum_{m=1}^{N}\left(\theta_{m}(\xi)\right)^{2}}+\sqrt{(1 / N) \sum_{m=1}^{N}\left(\hat{\theta}_{m}(\xi)\right)^{2}}\right)}, \\
& \operatorname{RMSE}=\frac{1}{N} \sqrt{\sum_{m=1}^{N}\left(\theta_{m}(\xi)-\widehat{\theta}_{m}(\xi)\right)^{2}} \\
& \mathrm{NSE}=\left\{1-\frac{\sum_{m=1}^{N}\left(\theta_{m}(\xi)-\widehat{\theta}_{m}(\xi)\right)^{2}}{\sum_{m=1}^{N}\left(\left(\theta_{m}(\xi)-\underline{\hat{\theta}}_{m}(\xi)\right)^{2}\right.}, \quad \widehat{\underline{\theta}}_{m}(\xi)=\frac{1}{N} \sum_{m=1}^{N} \hat{\theta}(\xi),\right. \\
& \mathrm{ENSE}=(1-\mathrm{NSE}),
\end{aligned}
$$

where $\theta_{m}$ is the analytical solution and $\widehat{\theta}_{m}$ represents the approximate solution by proposed algorithm. $N$ denotes the grid points.

\section{Numerical Simulation and Discussion}

In this section, different cases of (5) are considered to study the effect of variations in dimensionless end point load $(\rho)$ on large deflection of cantilever beam. The following cases are considered. Case I: $\rho=0.5$, Case II: $\rho=1.0$, Case III: $\rho=1.5$, and Case IV: $\rho=2.0$. The formulation of fitness functions for each case is given as follows:

$$
\text { Minimize } E=\frac{1}{N} \sum_{m=1}^{N}\left(\frac{\mathrm{d}^{2} \hat{\theta}}{\mathrm{d} \xi^{2}}+0.5\right)^{2}+\frac{1}{2}\left((\hat{\theta}(0)-0)^{2}+\left(\hat{\theta}^{\prime}(1)-0\right)^{2}\right) \text {, }
$$

$$
\text { Minimize } E=\frac{1}{N} \sum_{m=1}^{N}\left(\frac{\mathrm{d}^{2} \hat{\theta}}{\mathrm{d} \xi^{2}}+1.0\right)^{2}+\frac{1}{2}\left((\hat{\theta}(0)-0)^{2}+\left(\hat{\theta}^{\prime}(1)-0\right)^{2}\right) \text {, }
$$

Minimize $E=\frac{1}{N} \sum_{m=1}^{N}\left(\frac{\mathrm{d}^{2} \widehat{\theta}}{\mathrm{d} \xi^{2}}+1.5\right)^{2}+\frac{1}{2}\left((\hat{\theta}(0)-0)^{2}+\left(\hat{\theta}^{\prime}(1)-0\right)^{2}\right)$, 
TABLE 1: Setting of parameters for GNDO and SQP algorithm.

\begin{tabular}{|c|c|c|c|c|}
\hline Method & Parameters & Settings & Parameters & Settings \\
\hline \multirow{3}{*}{ GNDO } & Initialization & Random search & Bounds (Lb, Ub) & {$[-1,1]$} \\
\hline & Search agents & 70 & Max. iterations & 2000 \\
\hline & Function tolerance & $10^{-15}$ & Fitness limit & $10^{-15}$ \\
\hline \multirow{3}{*}{ SQP } & Initiation & Global best of GNDO & Bounds (Lb, Ub) & {$[-1,1]$} \\
\hline & Max. iterations & 1500 & $\mathrm{X}$-tolerance & $10^{-15}$ \\
\hline & Function tolerance & $10^{-15}$ & Fitness limit & $10^{-15}$ \\
\hline
\end{tabular}

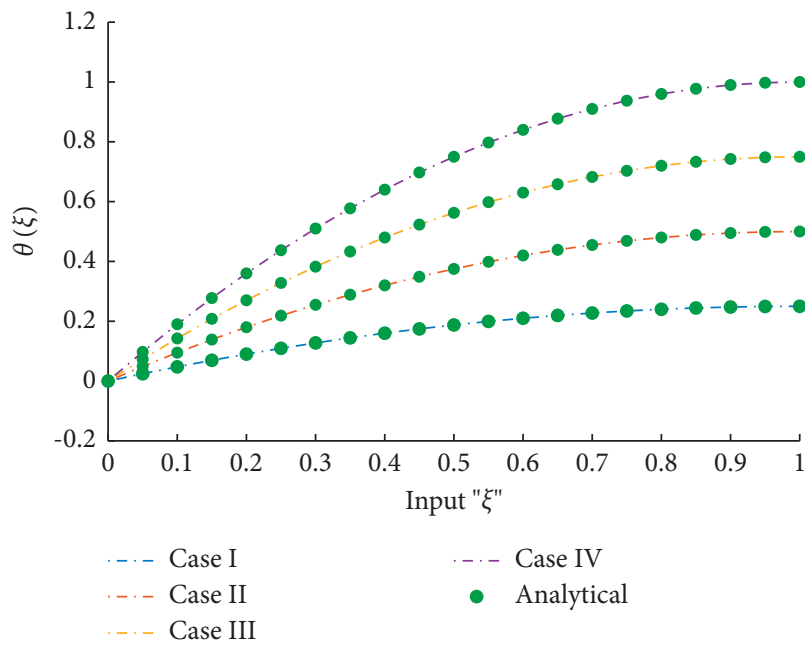

(a)

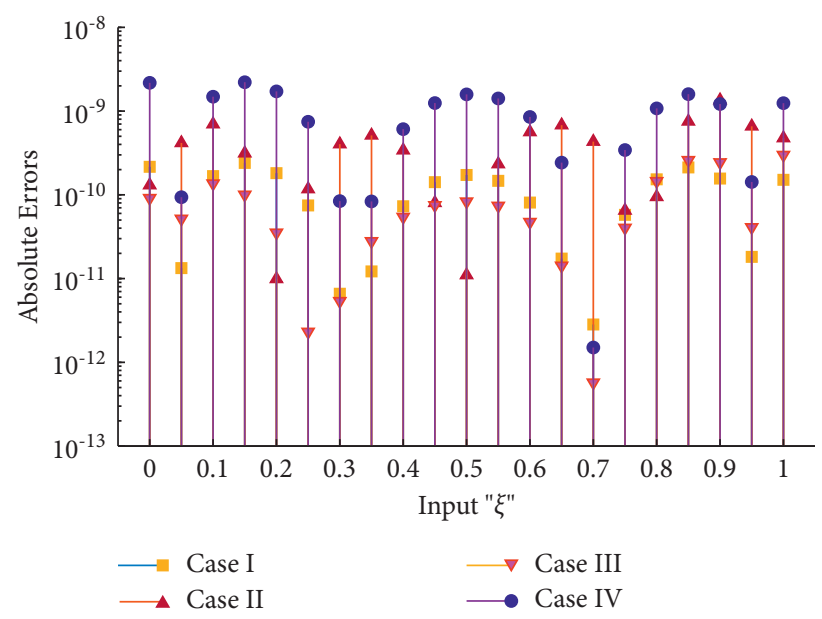

(b)

FIgURE 3: (a) Comparison of approximate solutions obtained by ANN-GNDO-SQP algorithm with analytical solution for each case. (b) Absolute errors in solutions of proposed algorithm for different cases.

$$
\begin{aligned}
\text { Minimize } E= & \frac{1}{N} \sum_{m=1}^{N}\left(\frac{\mathrm{d}^{2} \widehat{\theta}}{\mathrm{d} \xi^{2}}+2.0\right)^{2} \\
& +\frac{1}{2}\left((\hat{\theta}(0)-0)^{2}+\left(\hat{\theta}^{\prime}(1)-0\right)^{2}\right) .
\end{aligned}
$$

In this paper, the mathematical model of large deflected cantilever beam under end point load is investigated by developing soft computing technique. ANNs-based fitness function is constructed to model approximate solutions, which are further optimized by using hybridization of GNDO and SQP algorithms. To briefly study the efficiency and behaviour of proposed technique, it is executed for 100 times. Results obtained by ANN-GDDO-SQP algorithm are compared with exact solution and differential transformation method [33]. Approximate solutions along with absolute errors obtained by the proposed algorithm for different cases of deflected cantilever beam are shown in Figure 3. Figures 4(a) and 4(b) illustrate the influence of variations in dimensionless end point load $(\rho)$ on horizontal distance $\left(\delta_{h} / L\right)$ and rotational angle $\left(\theta_{\text {tip }}\right)$ of the beam at the tip or free end, respectively. Convergence of fitness values, MAD, TIC, RMSE, and ENSE for each case of large deflected cantilever beam during 100 independent runs is shown in Figures 5 and 6. Figure 7 represents the boxplots analysis for each case of deflected cantilever beam. The graphical illustration of mean absolute values, global values for fitness function, and performance indicators for each case study are presented in Figure 8.

It can be seen that the approximate solutions overlap the exact solution with minimum errors that show the accuracy of proposed technique. Table 2 dictates the comparison of approximate solutions and analytical solutions for different cases depending on variations in dimensionless point load at free end. Tables 3 and 4 show that absolute errors in best solutions for case I-IV lie around $1.51 E-10$ to $2.83 E-12,1.36 E-10$ to $5.72 E-13,1.35 E-$ 09 to $9.89 E-12$ and $1.08 E-09$ to $1.50 E-12$ with standard deviations $10^{-9}$ to $10^{-10}, 10^{-9}$ to $10^{-11}, 10^{-8}$ to $10^{-10}$ and $10^{-8}$ to $10^{-9}$, respectively. Table 5 dictates that mean or global values of fitness function for each case study are $1.80 E-08,5.18 E-08,1.10 E-07$, and $1.60 E-07$. Also, minimum values of MAD, TIC, RMSE, and ENSE lie around $10^{-6}, 10^{-7}, 10^{-6}$, and $10^{-10}$ with standard deviations around $1.80 E-05$ to $4.94 E-06,4.27 E-06$ to $1.59 E-06,1.50 E-05$ to $4.13 E-06$ and $2.35 E-08$ to $7.74 E-09$, respectively. Analyses based on the computational complexity of the design scheme for obtaining solution to equations (25)-(28) are dictated in Table 6 . Weights in ANN structure for best solution of each case obtained by proposed algorithm are presented in Table 7 and graphically shown in Figure 9. 


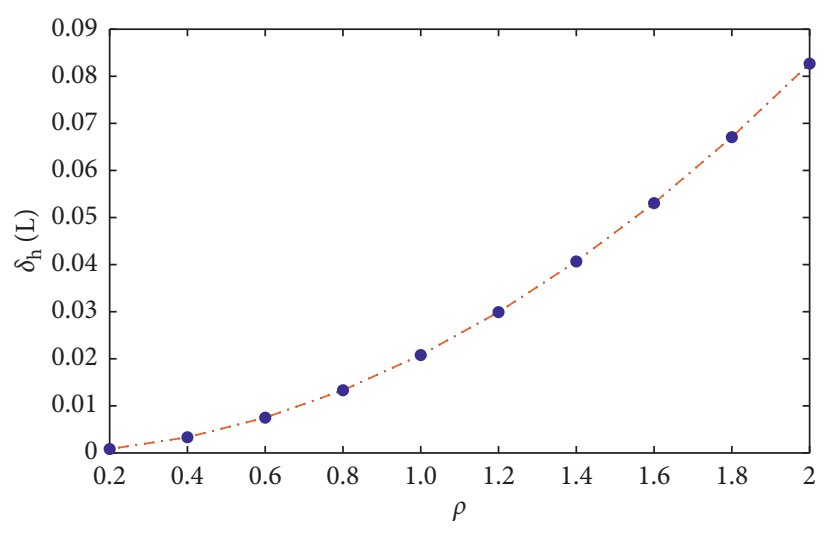

ANN-GNDO-SQP

- Analytical

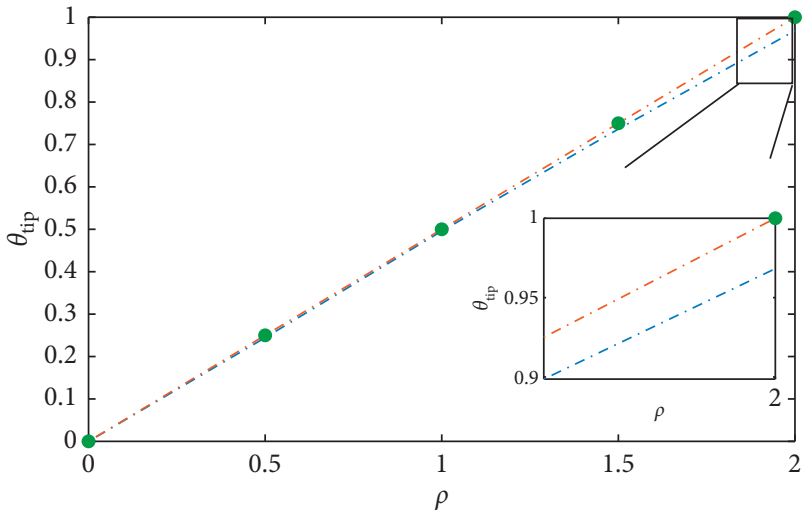

-.- DTM

-. - ANN-GNDO-SQP

- Analytical

(a)

(b)

FIgURe 4: Comparison between the (a) dimensionless horizontal distance and (b) rotational angle of the beam obtained by analytical method, DTM, and proposed algorithm for variations in dimensionless end-point load.

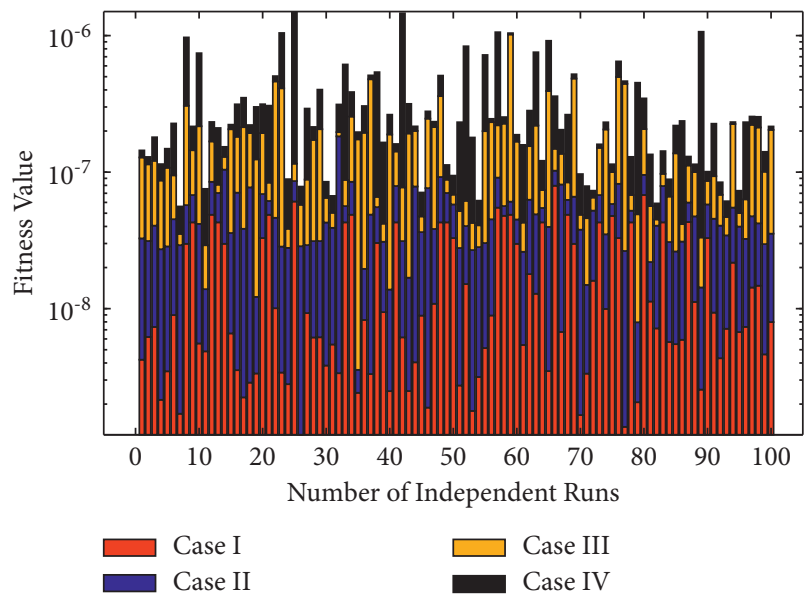

(a)

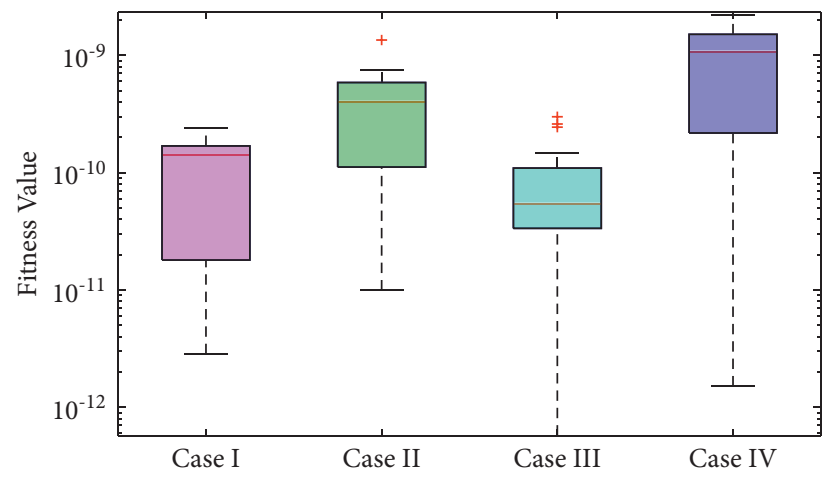

(b)

FIGURE 5: (a) Convergence of fitness value. (b) Box plot analysis for each case during 100 independent runs of ANN-GNDO-SQP algorithm.

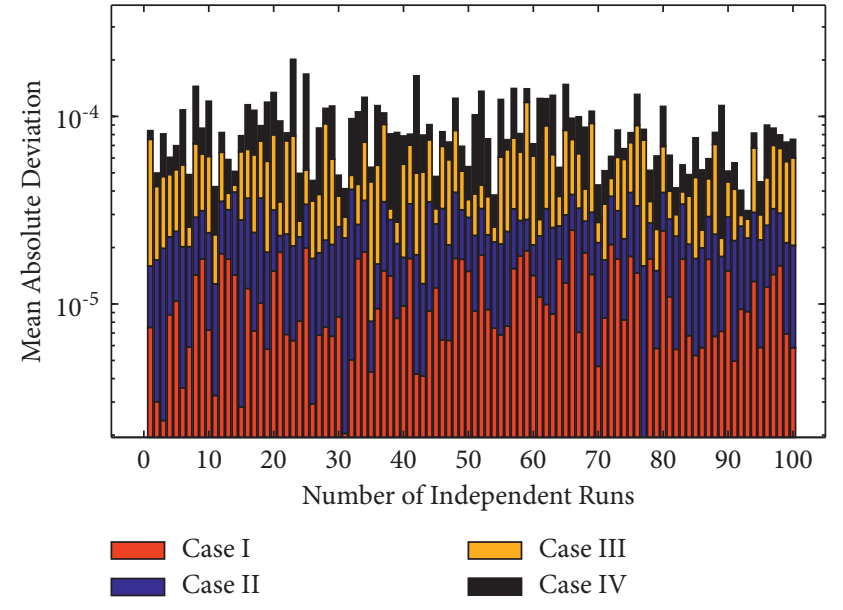

(a)

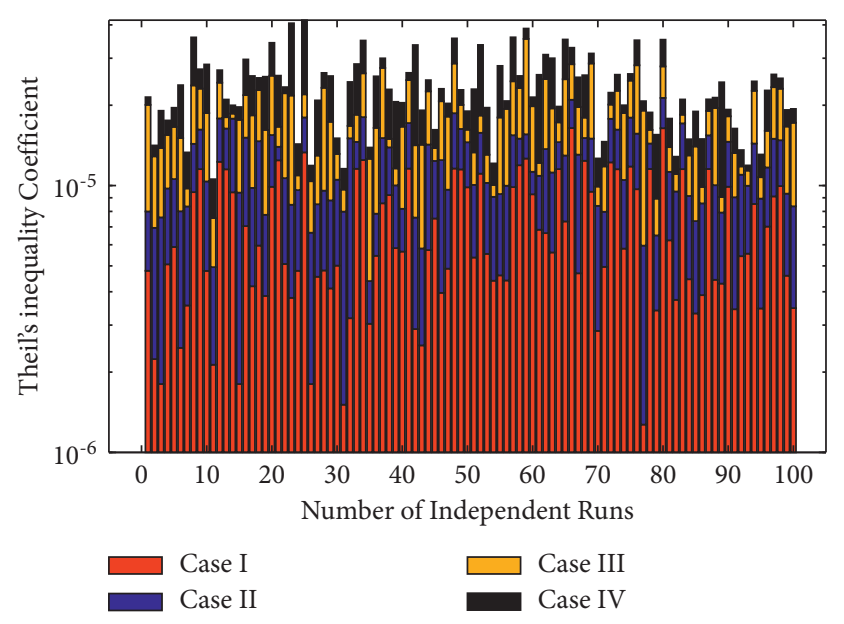

(b)

FIgUre 6: Continued. 


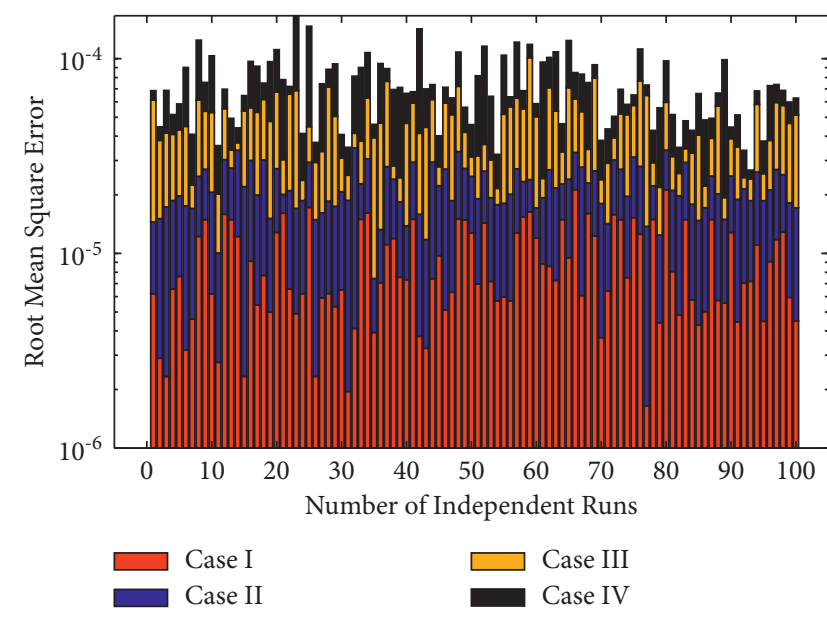

(c)

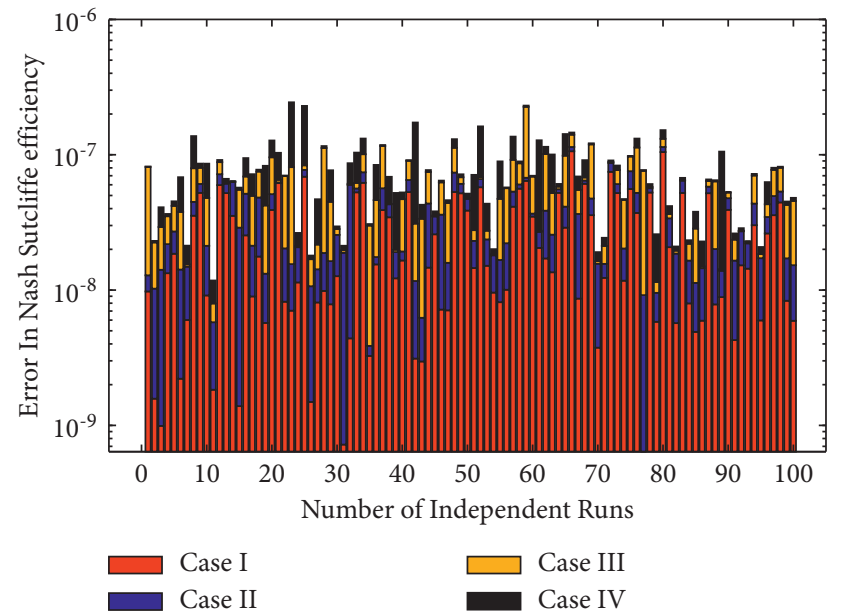

(d)

Figure 6: Convergence analysis of (a) MAD, (b) TIC, (c) RMSE, and (d) ENSE during 100 independent runs for each case of large deflected beam.

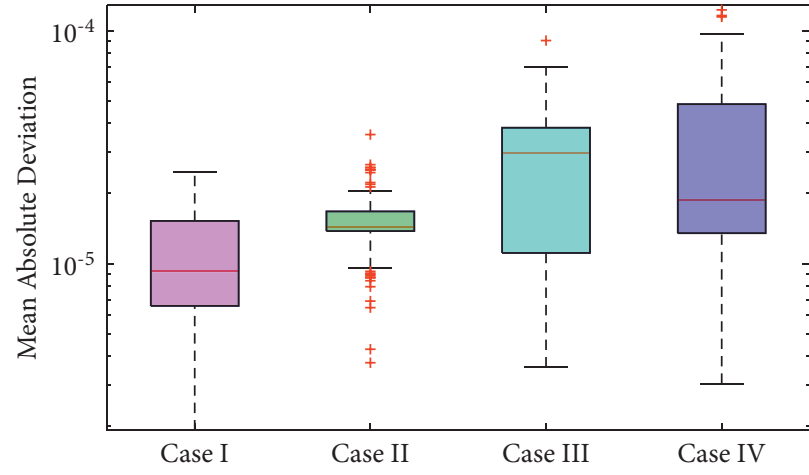

(a)

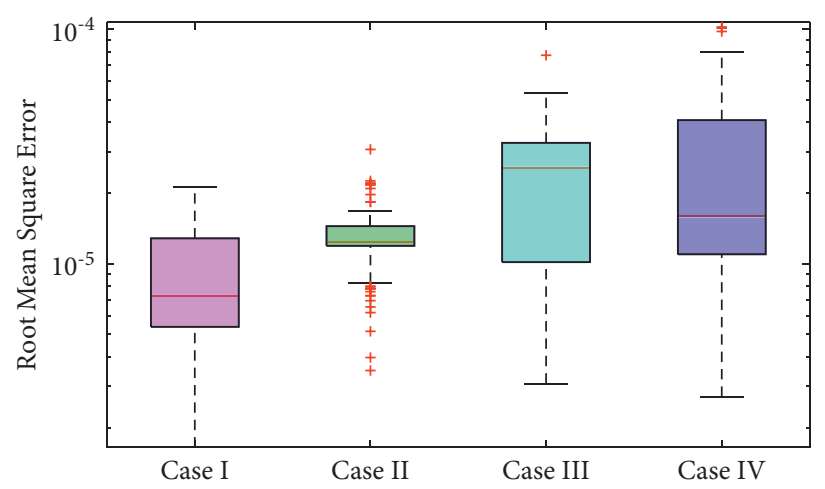

(c)

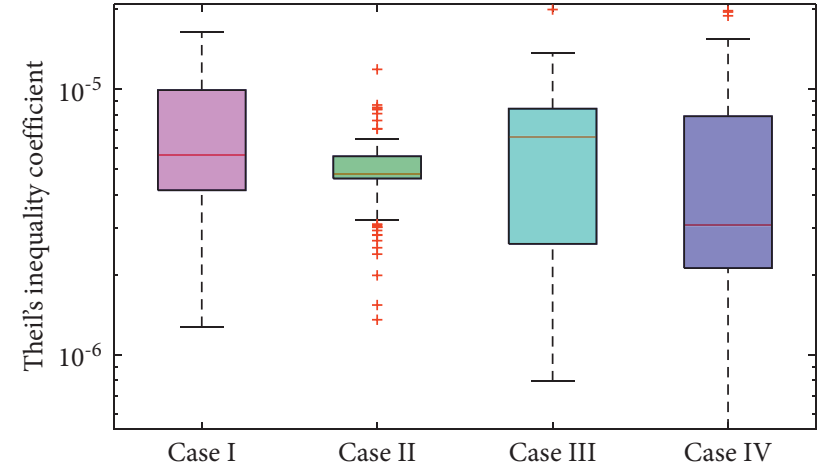

(b)

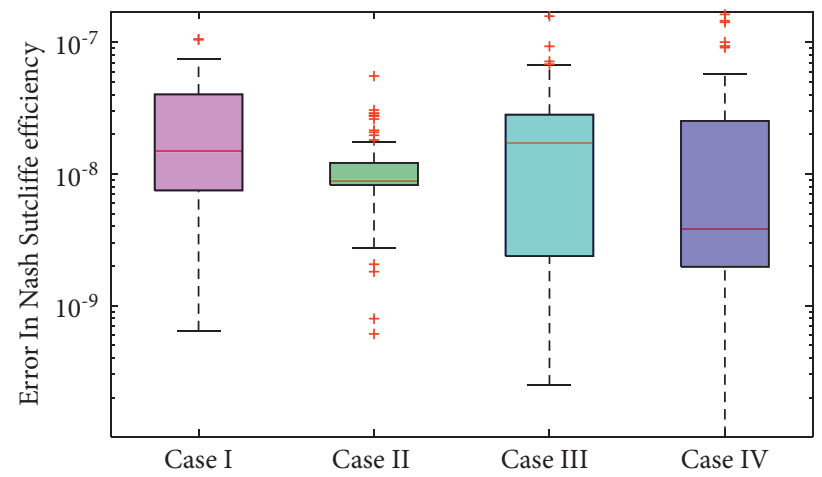

(d)

Figure 7: $(\mathrm{a}-\mathrm{d})$ represents the boxplot analysis for each case of large deflected cantilever beam under end-point load during 100 independent executions. (a) MAD, (b) TIC, (c) RMSE, and (d) ENSE. 


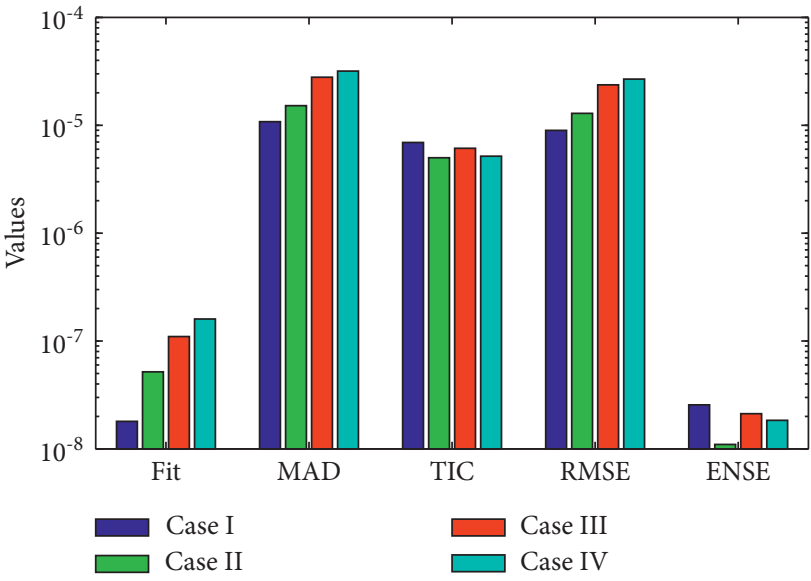

(a)

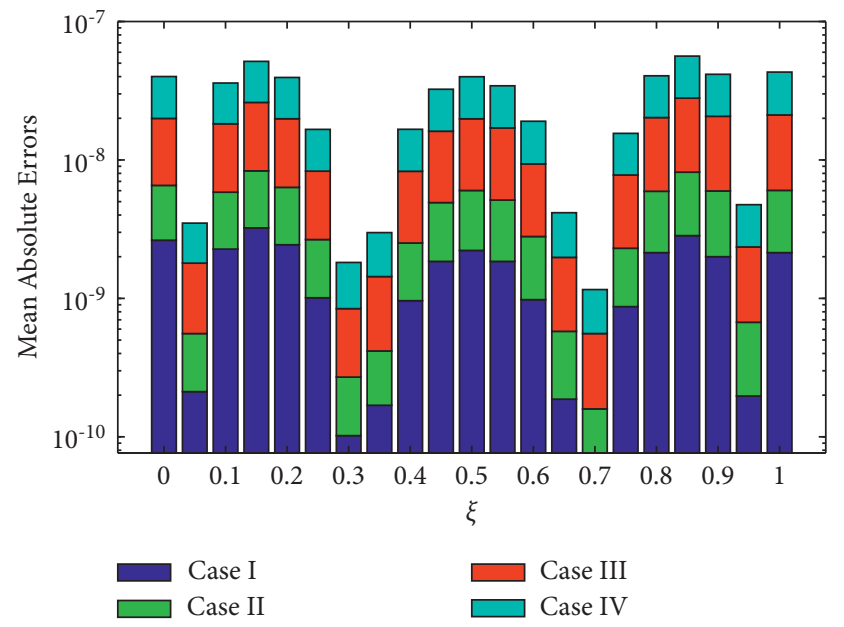

(b)

FIGURE 8: (a) The global values of fitness function and performance indicators obtained during multiple execution of ANN-GNDO-SQP algorithm. (b) Mean absolute errors in the solutions of proposed algorithm for different cases of large deflected cantilever beam.

TABLE 2: Comparison of the approximate solution obtained by ANN-GNDO-SQP algorithm with analytical solution for different variations is dimensionless end point load of large deflected cantilever beam.

\begin{tabular}{|c|c|c|c|c|c|c|c|c|}
\hline \multirow{2}{*}{$\xi$} & \multicolumn{2}{|r|}{$\rho=0.5$} & \multicolumn{2}{|r|}{$\rho=1.0$} & \multicolumn{2}{|r|}{$\rho=1.5$} & \multicolumn{2}{|r|}{$\rho=2.0$} \\
\hline & Exact & ANN-GNDO-SQP & Exact & ANN-GNDO-SQP & Exact & ANN-GNDO-SQP & Exact & ANN-GNDO-SQP \\
\hline 0.0 & 0 & $2.02 E-09$ & 0 & $-5.11 E-08$ & 0 & $-5.70 E-09$ & 0 & $6.07 E-07$ \\
\hline 0.1 & 0.0475 & 0.04750000 & 0.0950 & 0.09500000 & 0.1425 & 0.14250000 & 0.1900 & 0.19000004 \\
\hline 0.2 & 0.0900 & 0.09000000 & 0.1800 & 0.18000000 & 0.2700 & 0.27000000 & 0.3600 & 0.36000005 \\
\hline 0.3 & 0.1275 & 0.12750000 & 0.2550 & 0.25500000 & 0.3825 & 0.38250000 & 0.5100 & 0.51000000 \\
\hline 0.4 & 0.1600 & 0.16000000 & 0.3200 & 0.32000000 & 0.4800 & 0.48000000 & 0.6400 & 0.64000000 \\
\hline 0.5 & 0.1875 & 0.18750000 & 0.3750 & 0.37500000 & 0.5625 & 0.56250000 & 0.7500 & 0.75000008 \\
\hline 0.6 & 0.2100 & 0.21000000 & 0.4200 & 0.42000000 & 0.6300 & 0.63000364 & 0.8400 & 0.84000000 \\
\hline 0.7 & 0.2275 & 0.22750000 & 0.4550 & 0.45500000 & 0.6825 & 0.68250000 & 0.9100 & 0.91000000 \\
\hline 0.8 & 0.2400 & 0.24000188 & 0.4800 & 0.48000000 & 0.7200 & 0.72000000 & 0.9600 & 0.96000004 \\
\hline 0.9 & 0.2475 & 0.24750207 & 0.4950 & 0.49500000 & 0.7425 & 0.74250001 & 0.9900 & 0.99000006 \\
\hline 1.0 & 0.2500 & 0.25000192 & 0.5000 & 0.50000000 & 0.7500 & 0.75000000 & 1.0000 & 1.00000001 \\
\hline
\end{tabular}

TABLE 3: Maximum and minimum absolute errors obtained in ANN-GNDO-SQP solutions for different cases of large deflection of cantilever beam.

\begin{tabular}{|c|c|c|c|c|c|c|c|c|}
\hline \multirow{2}{*}{$\xi$} & \multicolumn{2}{|c|}{$\rho=0.5$} & \multicolumn{2}{|c|}{$\rho=1.0$} & \multicolumn{2}{|c|}{$\rho=1.5$} & \multicolumn{2}{|c|}{$\rho=2.0$} \\
\hline & Maximum AE & Minimum $\mathrm{AE}$ & Maximum AE & Minimum AE & Maximum $\mathrm{AE}$ & Minimum AE & Maximum AE & Minimum $\mathrm{AE}$ \\
\hline 0.0 & $1.07 E-08$ & $2.16 E-10$ & $2.54 E-08$ & $9.14 E-11$ & $1.28 E-07$ & $1.30 E-10$ & $1.24 E-07$ & $2.18 E-09$ \\
\hline 0.1 & $1.03 E-08$ & $1.67 E-10$ & $2.18 E-08$ & $1.36 E-10$ & $1.06 E-07$ & $6.97 E-10$ & $1.46 E-07$ & $1.48 E-09$ \\
\hline 0.2 & $1.02 E-08$ & $1.82 E-10$ & $2.46 E-08$ & $3.53 E-11$ & $1.27 E-07$ & $9.89 E-12$ & $1.41 E-07$ & $1.72 E-09$ \\
\hline 0.3 & $2.23 E-10$ & $6.59 E-12$ & $1.20 E-09$ & $5.38 E-12$ & $7.64 E-09$ & $4.03 E-10$ & $2.14 E-09$ & $8.42 E-11$ \\
\hline 0.4 & $4.76 E-09$ & $7.30 E-11$ & $8.77 E-09$ & $5.44 E-11$ & $4.32 E-08$ & $3.40 E-10$ & $8.23 E-08$ & $6.09 E-10$ \\
\hline 0.5 & $9.86 E-09$ & $1.73 E-10$ & $2.25 E-08$ & $8.30 E-11$ & $1.20 E-07$ & $1.10 E-11$ & $1.75 E-07$ & $1.58 E-09$ \\
\hline 0.6 & $3.79 E-09$ & $8.09 E-11$ & $1.11 E-08$ & $4.73 E-11$ & $6.36 E-08$ & $5.62 E-10$ & $7.28 E-08$ & $8.51 E-10$ \\
\hline 0.7 & $5.26 E-10$ & $2.83 E-12$ & $3.14 E-10$ & $5.72 E-13$ & $1.06 E-09$ & $4.32 E-10$ & $8.63 E-09$ & $1.50 E-12$ \\
\hline 0.8 & $1.02 E-08$ & $1.53 E-10$ & $2.09 E-08$ & $1.46 E-10$ & $1.14 E-07$ & $9.45 E-11$ & $2.06 E-07$ & $1.08 E-09$ \\
\hline 0.9 & $8.53 E-09$ & $1.56 E-10$ & $2.22 E-08$ & $2.44 E-10$ & $1.28 E-07$ & $1.35 E-09$ & $1.93 E-07$ & $1.22 E-09$ \\
\hline 1.0 & $1.04 E-08$ & $1.51 E-10$ & $2.07 E-08$ & $2.99 E-10$ & $1.20 E-07$ & $4.76 E-10$ & $2.30 E-07$ & $1.24 E-09$ \\
\hline
\end{tabular}




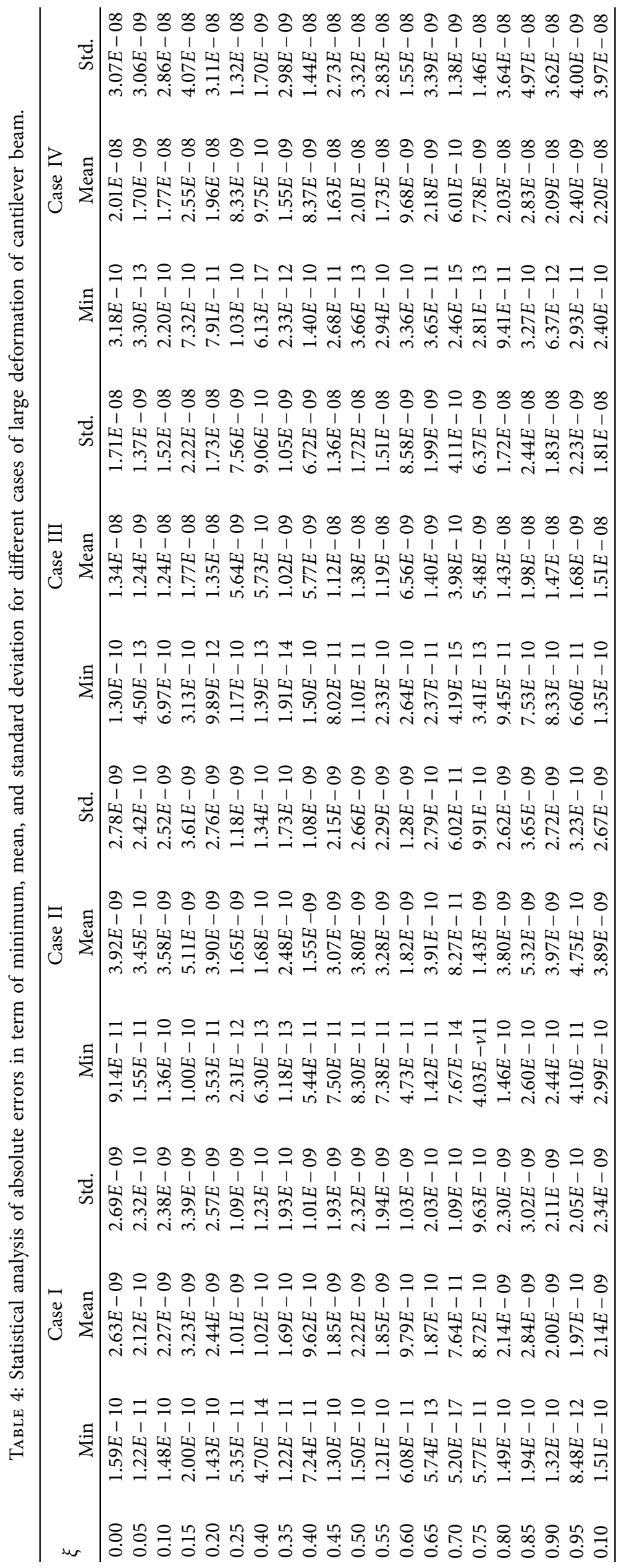




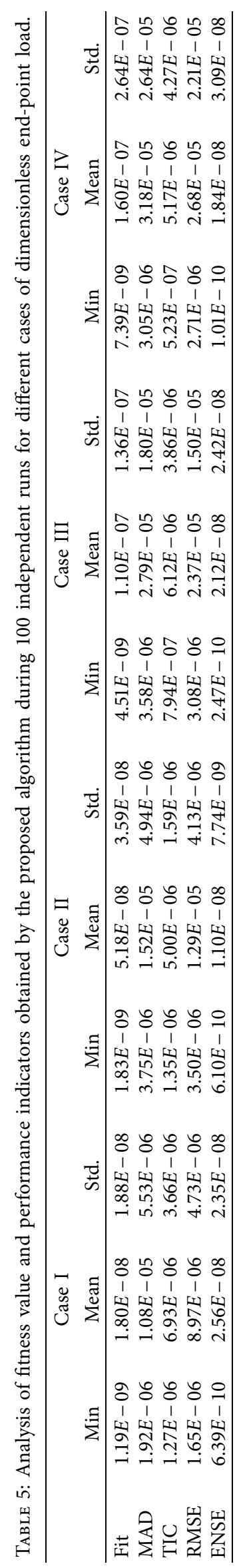




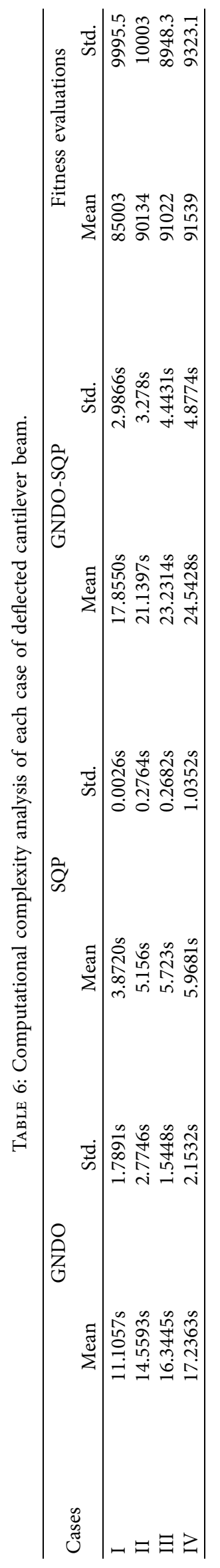




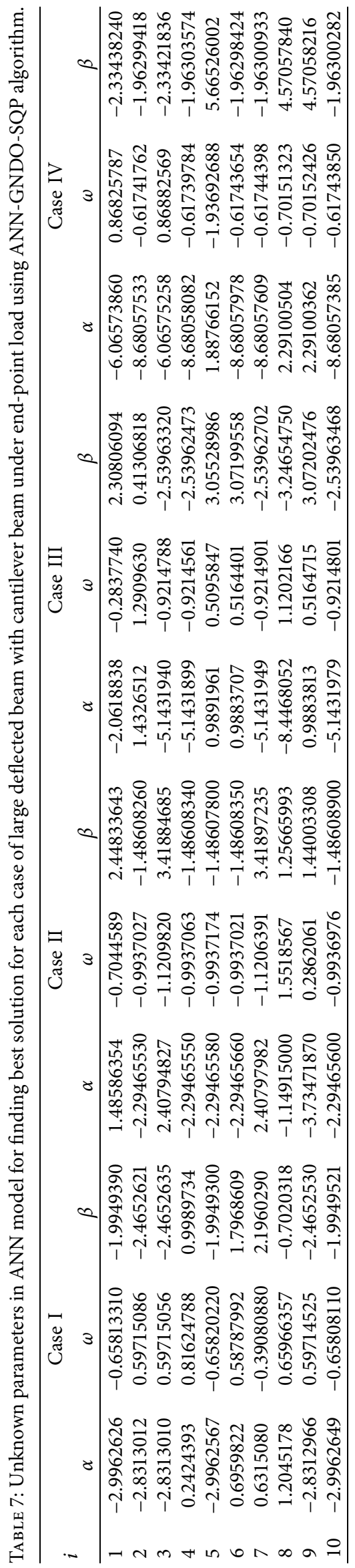




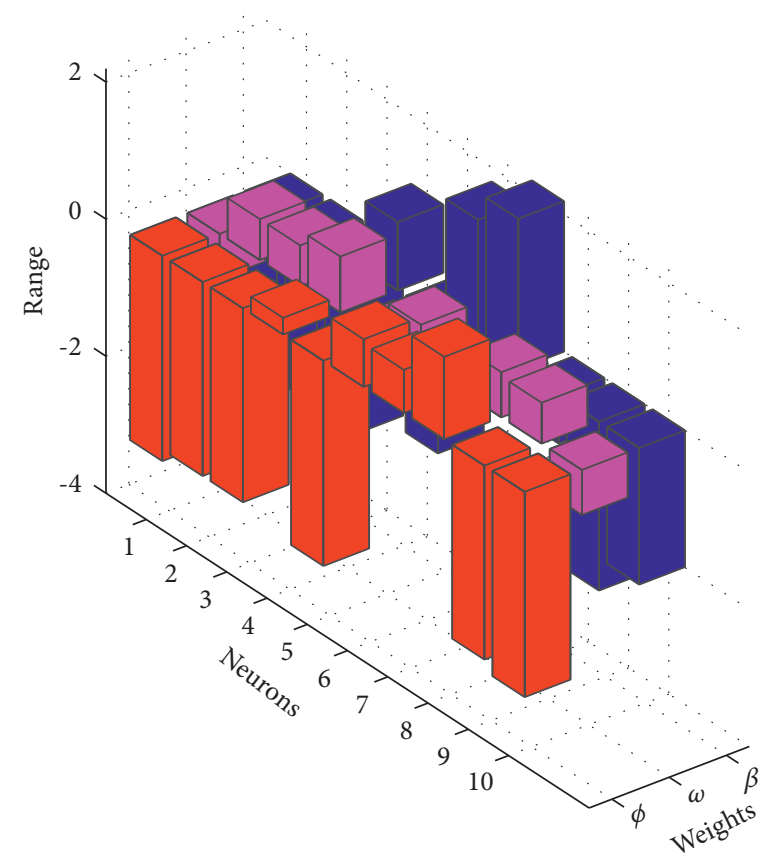

(a)

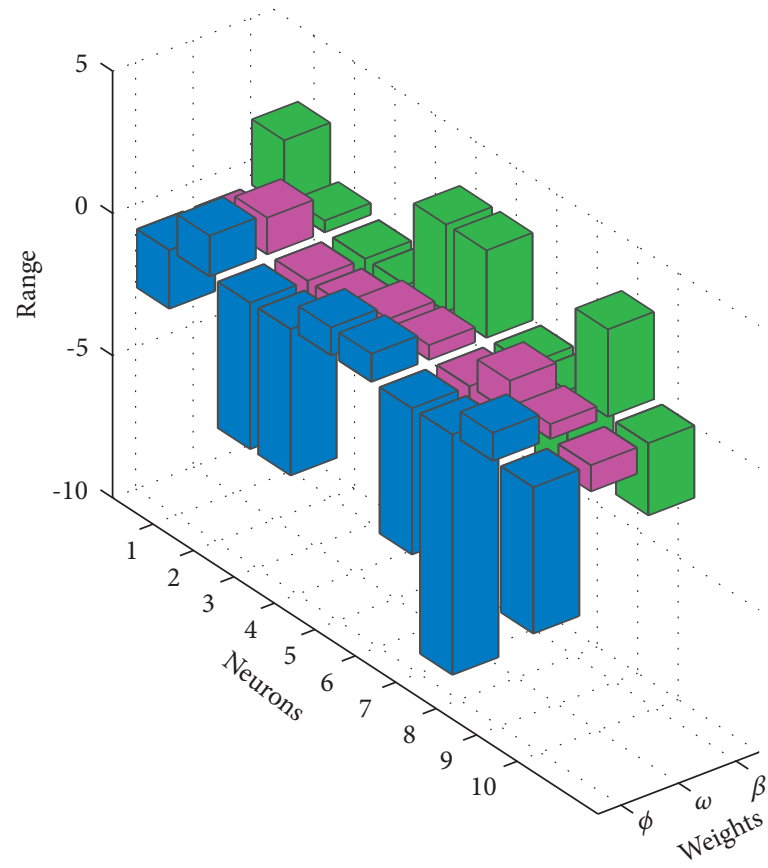

(c)

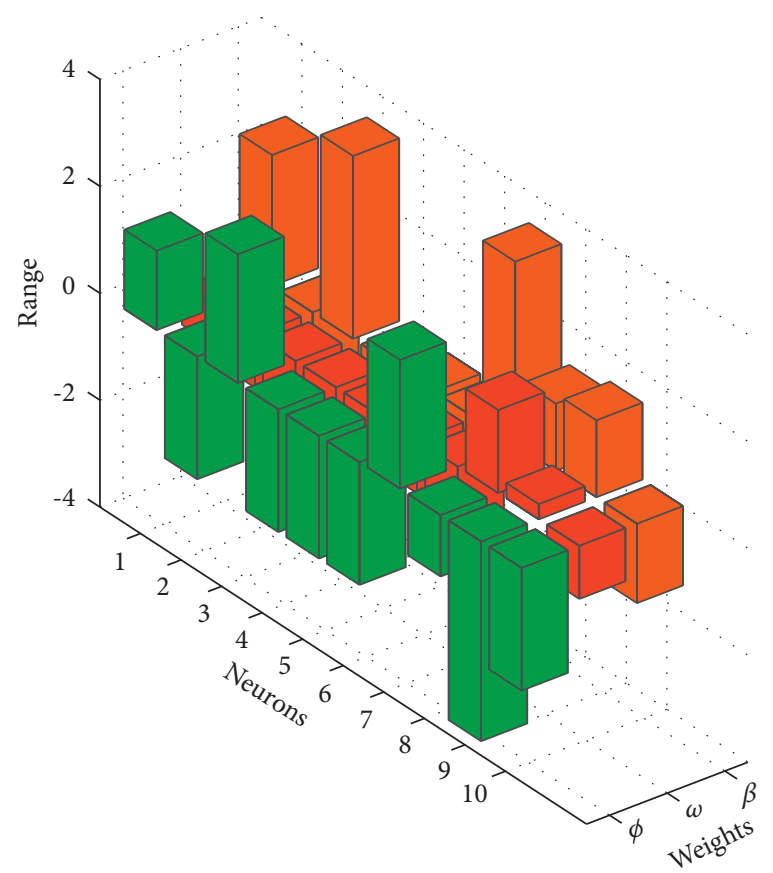

(b)

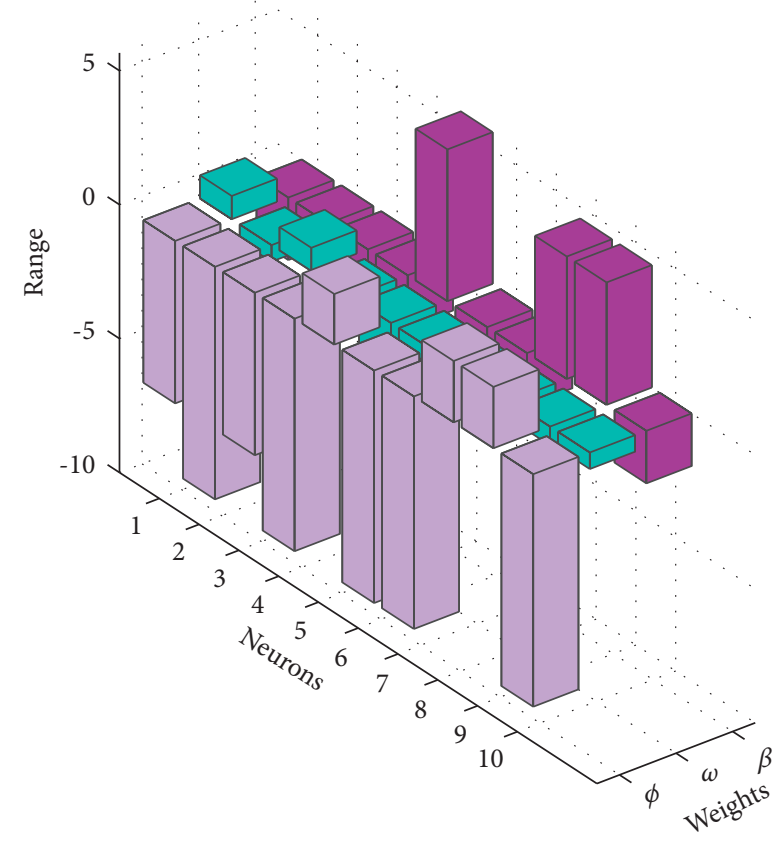

(d)

Figure 9: Unknown neurons in ANN structure corresponding to best solutions for each case large deflected cantilever beam. (a) Case I. (b) Case II. (c) Case III. (d) Case IV.

\section{Conclusion}

In this paper, we have analyzed the mathematical model of large deflected beam under variational point load and the free end. Furthermore, we present a new soft computing technique to calculate approximate solution for different cases depending on variations in end point load. We conclude our finding as follows. (i) A new soft computing evolutionary algorithm is developed in which the strength of artificial neural networks are utilized to model an approximate series solution and combined with hybridization of generalized normal distribution optimization algorithm and sequential quadratic programming. The proposed algorithm is named as ANN-GNDOSQP algorithm. 
(ii) ANNs-based fitness functions are constructed and optimize with the ANN-GNDO-SQP algorithm to obtain an overlapping solutions with minimum absolute errors as shown in Figure 3.

(iii) It can be seen that increasing end-point load increases the angle of deflection, horizontal distance, and rotational angle of the cantilever beam.

(iv) Convergence graphs and boxplots of 100 independent executions for fitness evaluation, MAD,
TIC, RMSE, and ENSE show the stability of proposed algorithm.

(v) Extensive graphical and statistical analysis along with complexity analysis of the proposed algorithm for solving large deflected cantilever beam shows the correctness and robustness of ANN-GNDO-SQP algorithm.

Approximate solutions for cases I-IV are as follows:

$$
\begin{aligned}
& \widehat{\theta}(\xi)\left\{\begin{array}{l}
\frac{-2.9962626}{1+e^{-(-0.65813310 t-1.9949390)}}+\frac{-2.8313012}{1+e^{-(0.59715086 t-2.4652621)},} \\
+\frac{-2.8313010}{1+e^{-(0.59715056-2.4652635)}}+\frac{0.2424393}{1+e^{(0.81624788 t+0.9989734)}}, \\
+\frac{-2.9962567}{1+e^{-(-0.65820220 t-1.9949300)}}+\frac{0.6959822}{1+e^{-(0.58787992 t+1.7968609)}}, \\
+\frac{0.6315080}{1+e^{-(-0.39080880 t+2.1960290)}}+\frac{1.2045178}{1+e^{(0.65966357 t-0.7020318)}}, \\
+\frac{-2.8312966}{1+e^{-(0.59714525 t-2.4652530)}}+\frac{-2.9962649}{1+e^{-(-0.65808110 t-1.9949521)}},
\end{array}\right. \\
& \widehat{\theta}(\xi)\left\{\begin{array}{l}
\frac{1.48586354}{1+e^{-(-0.7044589 t+2.44833643)}}+\frac{-2.29465530}{1+e^{-(-0.9937027 t-1.48608260)}}, \\
+\frac{2.40794827}{1+e^{-(-1.1209820+3.41884685)}}+\frac{-2.29465550}{1+e^{(-0.9937063 t-1.48608340)}} \\
+\frac{-2.29465580}{1+e^{-(-0.9937174 t-1.48607800)}}+\frac{-1.48607800}{1+e^{-(-0.9937021 t-1.48608350)}} \\
+\frac{2.40797982}{1+e^{-(-1.1206391 t+3.41897235)}}+\frac{-1.14915000}{1+e^{(1.5518567 t+1.25665993)}}, \\
+\frac{-3.73471870}{1+e^{-(0.2862061 t+1.44003308)}}+\frac{-2.29465600}{1+e^{-(-0.9936976 t-1.48608900)}},
\end{array}\right.
\end{aligned}
$$




$$
\hat{\theta}(\xi)\left\{\begin{array}{l}
\frac{-2.0618838}{1+e^{-(-0.2837740 t+2.30806094)}}+\frac{1.4326512}{1+e^{-(1.2909630 t+0.41306818)},} \\
+\frac{-5.1431940}{1+e^{-(-0.9214788-2.53963320)}}+\frac{-5.1431899}{1+e^{(-0.9214561 t-2.53962473)},} \\
+\frac{0.9891961}{1+e^{-(0.5095847 t+3.05528986)}}+\frac{0.9883707}{1+e^{-(0.5164401 t+3.07199558)}}, \\
+\frac{-5.1431949}{1+e^{-(-0.9214901 t-2.53962702)}}+\frac{-8.4468052}{1+e^{(1.1202166 t-3.24654750)}}, \\
+\frac{0.9883813}{1+e^{-(0.5164715 t+3.07202476)}}+\frac{-5.1431979}{1+e^{-(-0.9214801 t-2.53963468)}}, \\
\frac{-6.06573860}{1+e^{-(0.86825787 t-2.33438240)}}+\frac{-8.68057533}{1+e^{-(-0.61741762 t-1.96299418)},} \\
+\frac{-6.06575258}{1+e^{-(0.86882569-2.33421836)}}+\frac{-8.68058082}{1+e^{(-0.61739784 t-1.96303574)}}, \\
+\frac{1.88766152}{1+e^{-(-1.93692688 t+5.66526002)}}+\frac{-8.68057978}{1+e^{-(-0.61743654 t-1.96298424)}}, \\
+\frac{-8.68057609}{1+e^{-(-0.61744398 t-1.96300933)}}+\frac{2.29100504}{1+e^{(-0.70151323 t+4.57057840)}}, \\
+\frac{2.29100362}{1+e^{-(-0.70152426 t+4.57058216)}}+
\end{array}\right.
$$

\section{Abbreviations}

ANNs: $\quad$ Artificial neural networks

GNDO: Generalized normal distribution optimization

MAD: Mean absolute deviation

TIC: $\quad$ Theil's inequality coefficient

NSE: Nash-Sutcliffe efficiency

ENSE: $\quad$ Error in Nash-Sutcliffe efficiency

SQP: $\quad$ Sequential quadratic programming

RMSE: Root mean square error

DTM: Differential transform method

L: $\quad$ Distance of deflected beam

F: $\quad$ Concentrated load

$\phi_{n}, \omega_{n}, \beta_{n}$ : Unknown real valued neurons in ANNs

$\rho: \quad$ End-point load

$\kappa: \quad$ Curvature

$\theta: \quad$ Rotation of beam

$s: \quad$ Distance from fix point

I: $\quad$ Inertia

M: $\quad$ Bending moment

$E L: \quad$ Bending stiffness

$\delta_{h}: \quad$ Horizontal deflection of beam

$\xi: \quad$ Dimensionless parameter

$v_{i}^{t}: \quad$ Trail vector

$\widehat{\mu}_{i}: \quad$ Mean position

$\hat{\delta}_{i}: \quad$ Standard variance

$v_{i}^{t}: \quad$ Trail vector

$\hat{\mu}_{i}: \quad$ Mean position

$\widehat{\delta}_{i}: \quad$ Standard variance.

\section{Data Availability}

The data used to support the findings of this study are available from the corresponding author upon request.

\section{Conflicts of Interest}

The authors declare that they have no conflicts of interest.

\section{Acknowledgments}

This work was supported by the teaching research and reform project of Shanghai DianJi University (No. 6).

\section{References}

[1] M. A. Maneshi, E. Ghavanloo, and S. Ahmad Fazelzadeh, "Closed-form expression for geometrically nonlinear large deformation of nano-beams subjected to end force," The European Physical Journal Plus, vol. 133, no. 7, p. 256, 2018.

[2] C. M. Wang, Y. Xiang, and S. Kitipornchai, "Postbuckling of nano rods/tubes based on nonlocal beam theory," International Journal of Applied Mechanics, vol. 1, no. 2, pp. 259-266, 2009.

[3] D. Wang, B. Bienen, M. Nazem et al., "Large deformation finite element analyses in geotechnical engineering," Computers and Geotechnics, vol. 65, pp. 104-114, 2015.

[4] L. Monforte, M. Arroyo, J. M. Carbonell, and A. Gens, "Numerical simulation of undrained insertion problems in geotechnical engineering with the Particle Finite Element 
Method (PFEM)," Computers and Geotechnics, vol. 82, pp. 144-156, 2017.

[5] L. Monforte, M. Arroyo, J. M. Carbonell, and A. Gens, "Coupled effective stress analysis of insertion problems in geotechnics with the Particle Finite Element Method," Computers and Geotechnics, vol. 101, pp. 114-129, 2018.

[6] Y. Hu and M. F. Randolph, "H-adaptive FE analysis of elastoplastic non-homogeneous soil with large deformation," Computers and Geotechnics, vol. 23, no. 1-2, pp. 61-83, 1998.

[7] Y. Hu and M. F. Randolph, "A practical numerical approach for large deformation problems in soil," International Journal for Numerical and Analytical Methods in Geomechanics, vol. 22, no. 5, pp. 327-350, 1998.

[8] G. Qiu, S. Henke, and J. Grabe, "Application of a Coupled Eulerian-Lagrangian approach on geomechanical problems involving large deformations," Computers and Geotechnics, vol. 38 , no. 1, pp. 30-39, 2011.

[9] R. Schmidt and D. A. Dadeppo, "Approximate analysis of large deflections of beams," ZAMM-Zeitschrift für Angewandte Mathematik und Mechanik, vol. 51, no. 3, pp. 233-234, 1971.

[10] B. N. Rao and G. V. Rao, "On the large deflection of cantilever beams with end rotational load," ZAMM - Journal of Applied Mathematics and Mechanics/Zeitschrift für Angewandte Mathematik und Mechanik, vol. 66, no. 10, pp. 507-509, 1986.

[11] B. N. Rao and G. V. Rao, "Large deflections of a cantilever beam subjected to a rotational distributed loading," Forschung im Ingenieurwesen, vol. 55, no. 4, pp. 116-120, 1989.

[12] K. Lee, "Large deflections of cantilever beams of non-linear elastic material under a combined loading," International Journal of Non-Linear Mechanics, vol. 37, no. 3, pp. 439-443, 2002.

[13] S. Chucheepsakul and B. Phungpaigram, "Elliptic integral solutions of variable-arc-length elastica under an inclined follower force," Zamm, vol. 84, no. 1, pp. 29-38, 2004.

[14] L. Li, H. Tang, and Y. Hu, "The effect of thickness on the mechanics of nanobeams," International Journal of Engineering Science, vol. 123, pp. 81-91, 2018.

[15] M. Dado and S. Al-Sadder, "A new technique for large deflection analysis of non-prismatic cantilever beams," $\mathrm{Me}$ chanics research communications, vol. 32, no. 6, pp. 692-703, 2005.

[16] S. D. Akbas, "Forced vibration analysis of cracked functionally graded microbeams," Advances in Nano Research, vol. 6, p. 39, 2018.

[17] J. Wang, J.-K. Chen, and S. Liao, “An explicit solution of the large deformation of a cantilever beam under point load at the free tip," Journal of computational and applied mathematics, vol. 212, no. 2, pp. 320-330, 2008

[18] M. Khorasani, H. Elahi, M. Eugeni, L. Lampani, and O. Civalek, "Vibration of FG porous three-layered beams equipped by agglomerated nanocomposite patches resting on Vlasov's foundation," Transport in Porous Media, pp. 1-30, 2021.

[19] L. Li, Y. Hu, and X. Li, "Longitudinal vibration of size-dependent rods via nonlocal strain gradient theory," International Journal of Mechanical Sciences, vol. 115-116, pp. 135-144, 2016.

[20] R. Barretta, R. Luciano, F. Marotti de Sciarra, and G. Ruta, "Stress-driven nonlocal integral model for Timoshenko elastic nano-beams," European Journal of Mechanics-A/Solids, vol. 72, pp. 275-286, 2018.

[21] N. Tolou and J. Herder, "A seminalytical approach to large deflections in compliant beams under point load,"
Mathematical Problems in Engineering, vol. 2009, Article ID 910896, 13 pages, 2009.

[22] M. Mutyalarao, D. Bharathi, and B. N. Rao, "Large deflections of a cantilever beam under an inclined end load," Applied Mathematics and Computation, vol. 217, no. 7, pp. 3607-3613, 2010.

[23] N. A. Khan, M. Sulaiman, A. J. Aljohani, P. Kumam, and H. Alrabaiah, "Analysis of multi-phase flow through porous media for imbibition phenomena by using the LeNN-WOANM Algorithm," IEEE Access, vol. 8, pp. 196425-196458, 2020.

[24] W. Waseem, M. Sulaiman, A. Alhindi, and H. Alhakami, "A soft computing approach based on fractional order DPSO algorithm designed to solve the corneal model for eye surgery," IEEE Access, vol. 8, pp. 61576-61592, 2020.

[25] N. A. Khan, M. Sulaiman, P. Kumam, and A. J. Aljohani, "A new soft computing approach for studying the wire coating dynamics with oldroyd 8-constant fluid," Physics of Fluids, vol. 33, no. 3, p. 036117, 2021.

[26] Y. Zhang, J. Lin, Z. Hu, N. A. Khan, and M. Sulaiman, "Analysis of third-order nonlinear multi-singular emdenfowler equation by using the LeNN-WOA-NM Algorithm," IEEE Access, vol. 9, pp. 72111-72138, 2021.

[27] A. Ahmad, M. Sulaiman, A. Alhindi, and A. J. Aljohani, "Analysis of temperature profiles in longitudinal fin designs by a novel neuroevolutionary approach," IEEE Access, vol. 8, pp. 113285-113308, 2020.

[28] N. A. Khan, O. I. Khalaf, C. A. T. Romero, M. Sulaiman, and M. A. Bakar, "Application of euler neural networks with soft computing paradigm to solve nonlinear problems arising in heat transfer," Entropy, vol. 23, no. 8, p. 1053,2021 .

[29] W. Huang, T. Jiang, X. Zhang, N. A. Khan, and M. Sulaiman, "Analysis of beam-column designs by varying axial load with internal forces and bending rigidity using a new soft computing technique," Complexity, vol. 2021, Article ID 6639032, 19 pages, 2021.

[30] A. Ali, S. Qadri, W. Khan Mashwani et al., "Machine learning based automated segmentation and hybrid feature analysis for diabetic retinopathy classification using fundus image," Entropy, vol. 22, no. 5, p. 567, 2020.

[31] H. Wei, Q. X. Pan, O. B. Adetoro, E. Avital, Y. Yuan, and P. H. Wen, "Dynamic large deformation analysis of a cantilever beam," Mathematics and Computers in Simulation, vol. 174, pp. 183-204, 2020.

[32] P. Salehi, H. Yaghoobi, and M. Torabi, "Application of the differential transformation method and variational iteration method to large deformation of cantilever beams under point load," Journal of Mechanical Science and Technology, vol. 26, no. 9, pp. 2879-2887, 2012.

[33] H. Abbasi and A. Javed, "Implementation of differential transform method (DTM) for large deformation analysis of cantilever beam," IOP Conference Series: Materials Science and Engineering, vol. 899, Article ID 012003, 2020.

[34] Y. Zhang, Z. Jin, and S. Mirjalili, "Generalized normal distribution optimization and its applications in parameter extraction of photovoltaic models," Energy Conversion and Management, vol. 224, Article ID 113301, 2020.

[35] J. Nocedal and S. Wright, Numerical optimization, Springer Science \& Business Media, Berlin, Germany, 2006.

[36] K. Long, X. Wang, and X. Gu, "Multi-material topology optimization for the transient heat conduction problem using a sequential quadratic programming algorithm," Engineering optimization, vol. 50, no. 12, pp. 2091-2107, 2018. 
[37] A. Lang, Z. Song, G. He, and Y. Sang, "Profile error evaluation of free-form surface using sequential quadratic programming algorithm," Precision Engineering, vol. 47, pp. 344-352, 2017.

[38] Z. B. Sun, Y. Y. Sun, Y. Li, and K. P. Liu, "A new trust regionsequential quadratic programming approach for nonlinear systems based on nonlinear model predictive control," Engineering Optimization, vol. 51, no. 6, pp. 1071-1096, 2019.

[39] O. D. Montoya, W. Gil-González, and A. Garces, "Sequential quadratic programming models for solving the OPF problem in DC grids," Electric Power Systems Research, vol. 169, pp. 18-23, 2019.

[40] M. A. Z. Raja, M. A. Manzar, S. M. Shah, and Y. Chen, "Integrated intelligence of fractional neural networks and sequential quadratic programming for Bagley-Torvik systems arising in fluid mechanics," Journal of Computational and Nonlinear Dynamics, vol. 15, 2020.

[41] C.-L. Xiao and H.-X. Huang, "Optimal design of heating system in rapid thermal cycling blow mold by a two-step method based on sequential quadratic programming," International Communications in Heat and Mass Transfer, vol. 96, pp. 114-121, 2018. 